\title{
La competencia científico- tecnológica en la formación del futuro docente: algunos aspectos de la autopercepción con respeto a la integración de las TIC en el aula
}

Scientific-technological competence in the training of future teachers: self-perception regarding the integration of ICT in the classroom

\author{
Pedro TAdeu ${ }^{1}$ \\ ptadeu@ipg.pt \\ CI\&DEI-ESECD-Polytechnic of Guarda, Portugal
}

\section{Resumen:}

INTRODUCCIÓN. En la actualidad, se busca un modelo de profesorado competente, capaz de sortear los retos de la sociedad del conocimiento. Entre las competencias "deseables" destaca la científica y tecnológica, a fin de explorar e incluir todas las posibilidades ofrecidas por las Tecnologías de la Información y Comunicación $(\mathrm{TIC})$ en sus prácticas docentes. MÉTODO. El presente estudio responde a un diseño descriptivo-correlacional, cuya finalidad es analizar la percepción de los futuros docentes sobre su competencia científica en el uso de las TIC. Para ello, se elaboró un cuestionario ad hoc, de 30 ítems y cuatro opciones de respuestas. Las cuestiones incluían aspectos como su actitud hacia el uso de las TIC, qué importancia otorgaban a su inclusión en el aula, así como su opinión sobre si real-

\begin{abstract}
:
INTRODUCTION. We are currently looking for a competent teaching model able to face the new challenges of the knowledge society. Among the "desirable" competences a teacher should have, scientific and technological competences manifest themselves as of utmost importance since they are key to explore and include all the opportunities offered by Information and Communication Technologies (ICT) in their teaching practices. METHOD. This study adopted a descriptive-correlational design, whose purpose was to analyze the perception of future teachers about their scientific competence regarding the use of ICT. For this purpose, an ad hoc questionnaire was developed, with 30 items and four response options. The questions included aspects such as their attitude towards the use of ICT, what importance
\end{abstract}

1 Dirección para correspondencia (correspondence address):

Pedro Tadeu. CI\&DEI-ESECD-Polytechnic of Guarda. Avenida Dr. Francisco Sá Carneiro, n. ${ }^{\circ}$ 50. 6300-559 Guarda (Portugal). 
La competencia científico-tecnológica en la formación del futuro docente: algunos aspectos de la autopercepción con respeto a la integración de las TIC en el aula

Pedro Tadeu

mente estaban preparados para afrontar el reto de utilizarlas en su posterior ejercicio profesional. RESULTADOS. Los resultados revelan que los aspirantes a maestros se sienten tecnológicamente competentes, pero admiten que no son capaces de integrar las TIC en el aula pese a conocer las posibilidades didácticas que ofrecen. A su vez, los participantes poseen una valoración positiva hacia el uso de las TIC en el aula, aunque reconocen ciertas carencias formativas para asegurar su éxito cuando trabajen como maestros. Se establece una relación positiva entre la valoración de las TIC en la enseñanza, sus efectos en el aprendizaje, el conocimiento de su uso con fines didácticos y cómo incluirlas en las prácticas docentes. DISCUSIÓN. Se evidencia la necesidad de cambiar los programas formativos sobre tecnología educativa para alcanzar una integración equilibrada entre el conocimiento científico de las herramientas tecnológicas y el conocimiento didáctico-pedagógico.

\section{Palabras clave:}

Competencia científica; Educación Tecnológica; Profesores; Formación del profesorado; Educación Superior. they attached to the inclusion of ICT in the classroom, as well as their perception about whether they were really prepared to face the challenge of using them in their subsequent professional career. RESULTS. The results reveal that trainee teachers feel technologically competent, but admit that they are not able to integrate ICT into the classroom even though they know what teaching opportunities they offer. In turn, the participants have a positive appreciation of the use of ICT in the classroom, although they recognize a number of training needs to ensure their success when working as teachers. A positive relationship is established between the assessment of ICT in teaching, its effects on learning, knowledge of its use for teaching purposes and how to include it in their teaching careers. DISCUSSION. There is an evident need to reform training programs on educational technology in order to achieve a balanced integration between scientific knowledge of technological tools and didactic-pedagogical knowledge.

\section{Keywords:}

Scientific competence; Educational Technology; teachers; Teacher Education; Higher Education.

\section{Résumé}

INTRODUCTION. Actuellement, un modèle de professorat compétent, capable de surmonter les défis de la connaissance, est attendu. Parmi les compétences "souhaitables", les scientifique et technologique se démarquent, afin d'explorer et d'inclure toutes les possibilités offertes par les technologies de l'information et de la communication (TIC) dans ses pratiques d'enseignement. MÉTHODE. La présente étude répond à un modèle descriptif de correlation dont la finalité est d'analyser la perception des futurs enseignants quant à leur compétence scientifique dans I'utilisation des TIC. Pour cela, un questionnaire ad hoc de 30 items avec quatre options de réponses a été élaboré. Les questions portaient sur des aspects tels que leur attitude envers I'utilisation des TIC, I'importance accordée à leur intégration en classe, ou leur opinion sur leur préparation pour affronter le défis de les utiliser dans leur futur exercice professionnel. RÉSULTATS. Les résultats révèlent que les futurs enseignants se sentent technologiquement compétents, mais admettent qu'ils ne sont pas capables d'intégrer les TIC en classe, même s'ils reconnaissent les possibilités didactiques qu'elles offrent. De même, les participants ont une appréciation positive envers l'utilisation des TIC en classe, bien qu'ils reconnaissent quelques lacunes formatives pour assurer son succès quand ils travailleront comme professeurs. Une relation positive entre l'appréciation des TIC dans l'enseignement, leurs effets sur I'apprentissage, la connaissance de leur utilisation à des fins didactiques et comment les intégrer à la pratique d'enseignement est établie. DISCUSSION. La nécessité de changer les programmes de formation en technologie éducative pour atteindre une intégration équilibrée entre la connaissance scientifique des outils technologiques et la connaissan- 
La competencia científico-tecnológica en la formación del futuro docente: algunos aspectos de la autopercepción con respeto a la integración de las TIC en el aula

ce didactico-pédagogique est manifeste.

\section{Mots clés:}

Compétence scientifique; éducation technologique; professeurs; formation des enseignants; éducation supérieure.

Fecha de recepción: 08-02-2020

Fecha de aceptación: 12-09-2020

\section{Introducción}

En las últimas décadas, la formación del profesorado ha sufrido un vertiginoso avance en cuanto a la competencia científica (Sánchez-Prieto, Hernández-García, García-Peña, Chaparro-Peláez y Olmos-Migueláñez, 2019). Este hecho se atisba en la esencial formación y conocimiento de las Tecnologías de la Información y el Conocimiento (TIC) para su puesta en práctica en los centros escolares e institutos (García-Pérez, RebolloCatalán y García-Pérez, 2016).

La formación en competencias es un aspecto crucial en el nuevo enfoque universitario desde la convergencia del Espacio Europeo de Educación Superior, hace más de una década (López, Pérez-García y Rodríguez, 2015). Se demandan profesionales competentes, que sean capaces de asumir los retos de la sociedad del conocimiento y la volatilidad y rapidez de los cambios que acontecen fuera de las aulas (De-Juanas Oliva, Martín del Pozo y González-Ballesteros, 2016).

En el caso de la formación docente, esta necesidad se agudiza considerablemente, pues no solo se trata de atender a un alumnado diverso (Fernández-Batanero, Cabero y López-Meneses, 2018), sino que además, hay que hacer un buen uso de los recursos disponibles, especialmente los tecnológicos, para diseñar procesos pedagógicos significativos y estimulantes para ellos (Liu, Wu y Chen, 2013).

\section{Marco teórico}

La irrupción de las nuevas tecnologías y la web 2.0 ha supuesto un cambio paradigmático en torno a cómo se desarrollan los procesos educativos. Tal es el caso, que se observa una necesidad emergente de capacitar al profesorado en el conocimiento y uso de las TIC (Magen-Nagar 
La competencia científico-tecnológica en la formación del futuro docente: algunos aspectos de la autopercepción con respeto a la integración de las TIC en el aula Pedro Tadeu

y Shonfeld, 2018). Agencias y organismos internacionales se han hecho eco de tal necesidad, desarrollando proyectos y programas específicos destinados a la capacitación tecnológica docente (Gabarda-Méndez, Rodríguez-Martín y Moreno-Rodríguez, 2017). Ejemplo de ello es el proyecto "Aprovechar las TIC para alcanzar las metas de Educación 2030", diseñado por el Fondo UNESCO-Grupo Weidong, cuya finalidad es contribuir a la formación docente en TIC, en aras de alcanzar el Objetivo de Desarrollo Sostenible (ODS) en 2030, a través de una educación inclusiva y equitativa de calidad.

Hablar de competencia científica (docente), supone hablar de un conjunto de habilidades y destrezas que el profesorado pone en juego en virtud de sacar el máximo rendimiento en el alumnado (Casey, Goodyear y Armor, 2017). Cuando la competencia científica viene supeditada al uso y conocimiento (didáctico) de recursos tecnológicos, estaríamos hablando de competencia digital (docente). Esta podría definirse como el conjunto de destrezas y conocimientos necesarios para desenvolverse en la Era digital que caracteriza la Sociedad de la Información. Ferrari (2013) identifica 5 áreas de actuación en los que se desarrolla la competencia digital: (a) información, (b) comunicación, (c) creación de contenido, (d) seguridad y (e) resolución de conflictos. Desde este prisma, parece que la competencia digital equivale a un amplio conjunto de conocimientos, habilidades y destrezas que afectan no solo al dominio de la tecnología, si no al uso ejercido sobre esta y en su potencial para atender a los retos de la sociedad actual. Al respecto, autores como Gisbert-Cervera, González-Martínez y Esteve-Mon (2016) sostienen que la competencia digital docente debe situarse en una encrucijada, en la que ya superadas dicotomías como nativo e inmigrante digital, es tiempo de buscar fórmulas encaminadas a que el profesorado "sea competente" en el uso educativo de la tecnología. Es importante clarificar algunos términos que, en la práctica suelen utilizarse indistintamente, a pesar de tener connotaciones diferentes en su origen. Así, la alfabetización tecnológica alude al manejo de la tecnología, es decir al conocimiento de las habilidades digitales básicas. En cierto modo, supone el nivel más superficial de conocimiento técnico. El fin en sí mismo, desde esta concepción, es dominar los nuevos procedimientos y recursos digitales, tras la digitalización de la enseñanza y el aprendizaje. Desde aquí, no entra en escena el conocimiento pedagógico, ni el uso didáctico que se hace de los mismos, tal y como se analizan en modelos de orden superior 
como el TPACK (Koehler, Mishra y Cain, 2015). A diferencia del anterior, la competencia tecnológica integra los saberes relacionados con la tecnología, es decir, por un lado supone el conocimiento instrumental de los medios y recursos tecnológicos, de las posibilidades de comunicación y de actuación de los dispositivos tecnológicos y su uso adecuado para un propósito definido (Falcó Boudet, 2017).

Una vez clarificados competencia digital, alfabetización tecnológica y competencia tecnológica como conceptos y procesos circunscritos a la incorporación de las TIC en el aula, es momento de examinar las cuestiones que han de ser resueltas, para lograr su efectividad en el aula. El primer interrogante que surge es si realmente el profesorado está cualificado para afrontar los retos que supone una verdadera inclusión de las TIC en la escuela (Fernández-Batanero, Sañudo, Montenegro-Rueda y García-Martínez, 2019). No solamente se trata de conocer la tecnología, más bien el interrogante planteado radica en cómo usarla correctamente con fines pedagógicos (Khine, Afari y Ali, 2019). Precisamente, sobre esta cuestión, han surgido numerosas investigaciones que han llegado a la conclusión de que el profesorado valora positivamente el uso de las TIC (Sánchez-García y Galindo-Villardón, 2018), aunque manifiesta no tener un conocimiento suficiente en aprovechar todas las posibilidades reales que les brinda (George y Sanders, 2017), debido a una escasa formación durante su periodo en la universidad.

Específicamente en la Educación Superior, existen diversas investigaciones que han puesto el foco sobre la capacitación científico-tecnológica de los docentes en formación (García-Valcárcel Muñoz-Repiso y Martín del Pozo, 2016; Gallego-Arrufat, Gámiz-Sánchez y GutiérrezSantiuste, 2010). Ejemplo de ello es la investigación desarrollada por Martínez, Leite y Monteiro (2016), en la que analizaron la idoneidad de la formación en TIC en estudiantes de magisterio. Entre sus resultados hallaron que existen espacios destinados a la formación en TIC, pero que éstos tienden a ser insuficientes o a abordar aspectos carentes de sentido para el posterior desempeño docente. Este hallazgo ha sido reiterado en numerosas investigaciones halladas en todo el mundo. De igual forma, la competencia científica-tecnológica ha sido relacionada con factores como la mejora escolar, la motivación del alumnado y la inclusión (Hersh, 2017). Si el objetivo de la educación estriba en ofrecer una enseñanza de calidad, que redunde en aprendizajes significativos construidos por el alumnado, es preciso iniciar procesos de reflexión so- 
bre la profesión docente, incluyendo todas las herramientas necesarias para lograrlo.

En este sentido, la presente investigación aborda esta cuestión, tratando de ofrecer una mirada del caso portugués sobre la formación en competencias científico-tecnológica en estudiantes adscritos a titulaciones de enseñanza básica.

\section{Método}

\section{Objetivos de la investigación}

El presente estudio descriptivo-correlacional responde a tres objetivos de investigación, que son:

- Conocer la percepción del estudiante en formación sobre el uso de las TIC.

- Identificar el grado de adquisición de la competencia científica de los futuros docentes.

- Analizar el nivel de utilización de las TIC de los futuros docentes en su periodo de prácticas.

\section{Participantes}

La muestra está compuesta por 107 estudiantes de primer curso (71 mujeres y 36 hombres) de enseñanza básica que estaban matriculados en el Instituto Politécnico da Guarda ( $M=20,86$ y DT=2,27). Los estudiantes estaban cursando la materia de Educación Multimedia, en la que se examinaban diferentes recursos tecnológicos y herramientas didácticas en el transcurso de las clases. A todos los estudiantes se les proporcionó el consentimiento informado aceptando la participación en el estudio asegurando su total anonimato.

\section{Instrumento}

Se elaboró un cuestionario específico para esta investigación compuesto por 30 ítems con una escala de respuesta tipo Likert. Las cuatro opciones de respuesta comprendían 1 "nada", 2 "poco", 3 "bastante" y 4 "mucho". Los ítems abordan cuestiones relacionadas con la formación 
acerca del uso de las TIC, su utilización en el aula, la percepción sobre el impacto de las TIC en el proceso de enseñanza-aprendizaje e incluyen una valoración de los participantes hacia las TIC. En el cuestionario, se incluyeron ítems sobre algunos de los recursos, herramientas y plataformas estudiadas en clase, tales como las wikis o webquest.

\section{Procedimiento}

La escala se administró dentro del horario escolar, utilizando la herramienta de Google Form. En todo momento, los profesores estuvieron presentes y ayudaron a los estudiantes con cualquier pregunta. Se aseguró a los estudiantes que sus respuestas se mantendrían en el anonimato y que toda la información proporcionada durante el curso del estudio se utilizaría únicamente con fines científicos.

\section{Análisis de los datos}

Para el análisis de los datos se utilizó el Software SPSS v.24 para el cálculo de los descriptivos y el análisis factorial para los datos descriptivos y el AMOS para el análisis exploratorio.

Se utilizó el análisis de frecuencias relativas para describir la percepción del estudiante en formación sobre el uso de las TIC e identificar el grado de adquisición de competencia científica de los futuros profesores. Además, para realizar el cruce de las variables, se aplicó el test de independencia chi-cuadrado, que permitió identificar posibles asociaciones entre las variables que determinan el nivel de uso de las TIC de los futuros profesores en su periodo de práctica. La aplicación de la prueba de chi-cuadrado presupone muestras superiores a 20 elementos y que al menos el $80 \%$ de las frecuencias esperadas son superiores a 5 . Si una de las suposiciones no se cumple, se utiliza la prueba exacta de Fisher utilizando un nivel de significación del 5\%.

\section{Resultados}

Para la elaboración de los resultados, se efectuó el cálculo de frecuencias de los ítems del cuestionario y se establecieron correlaciones entre los ítems relacionados, a fin de dar respuesta a los objetivos de la investigación. 
La competencia científico-tecnológica en la formación del futuro docente: algunos aspectos de la autopercepción con respeto a la integración de las TIC en el aula Pedro Tadeu

De esta forma, la tabla 1 incluye la frecuencia de las respuestas de los 107 participantes para cada ítem. Así, se observa que la mayoría de los encuestados admiten que han cursado asignaturas relacionadas con las TIC, oscilando su tasa de respuesta entre mucho $(92,5 \%)$ y bastante $(7,5 \%)$. No obstante, a pesar de que la mayoría han cursado materias relacionadas con el uso de las TIC, cuando se les preguntó por su percepción sobre la importancia de usar las NNTT en el aula, los participantes consideraron que no eran especialmente importantes, concentrándose sus respuestas entre poco $(72.9 \%)$ y bastante $(23,4 \%)$.

A pesar de afirmar conocer las posibilidades didácticas de las TIC (aproximadamente el 80\%), su tasa de respuesta decrece notablemente al preguntarles si la integración de las TIC, en términos de su incorporación en los procesos instructivos, favorece el aprendizaje (en torno al 55\% de los participantes afirman bastante/mucho) y sensiblemente cuando se les pregunta por la tendencia de uso del profesorado universitario (sobre el $76 \%$ consideran que entre bastante y mucho).

Al preguntarles si se sienten competentes tecnológicamente, la mayoría afirma serlo, aunque se aprecian distintos niveles ( $\mathrm{Nada}=6,5 \%$; Poco $=37,4 \%$; Bastante $=31,8 \%$; Mucho $=24,3 \%$ ). Paradójicamente, sus respuestas son sustancialmente inferiores cuando se les preguntan si se sienten preparados para usar las TIC ( $\mathrm{Nada}=26,2 \%$; PoCO $=43 \%$; Bastante $=29,9 \%$; Mucho=0,9\%) o por la formación recibida sobre TIC y su uso cuando sean maestros ( $\mathrm{Nada}=13,1 \%$; $\mathrm{Poco}=42,1 \%$; Bastante $=35,5 \%$; Mucho $=9,3 \%$ ).

A su vez, relacionan las TIC con la innovación docente (Nada=3,7\%; Poco $=23,4 \%$; Bastante $=45,8 \%$; Mucho $=27,1 \%$, considerando importante que los docentes estén alfabetizados tecnológicamente $(\mathrm{Nada}=0,9 \%$; Poco=29,0\%; Bastante=44,9\%; Mucho=25,2\%), aunque señalan la complejidad de la integración efectiva de las TIC en el aula (Poco=12,1\%; Bastante=20,6\%; Mucho=67,3\%).

Respecto a la atención a la diversidad, los participantes afirman que las TIC son adecuadas para los procesos de enseñanza y aprendizaje del alumnado con necesidades educativas especiales (Poco=18,7\%; Bastante $=19,6 \%$; Mucho $=61,7 \%$ ), sentenciando su idoneidad para lograr la inclusión (Bastante=54,2\%; Mucho=45,8\%).

En paralelo, al solicitarles una valoración general sobre el uso de las herramientas tecnológicas en la escuela y de la formación del profesorado, en ambas cuestiones las respuestas se situaron entre poco y bastante; 
La competencia científico-tecnológica en la formación del futuro docente: algunos aspectos de la autopercepción con respeto a la integración de las TIC en el aula

mientras que se halla unanimidad a la hora de afirmar su utilidad en educación (Poco=8,4\%; Bastante=30,8\%; Mucho=60,7\%).

Tabla 1

Frecuencia de los ítems del cuestionario ad hoc

Ítem

Nada Poco Bastante Mucho

¿Ha cursado asignaturas sobre TIC?

$0 \% \quad 0 \% \quad 7,5 \% \quad 92,5 \%$

¿Considera que es importante el uso de las

NNTT en el aula?

$3,7 \% \quad 72,9 \% \quad 23,4 \% \quad 0 \%$

¿El profesorado universitario utiliza las TIC en

el aula?

$6,5 \% \quad 17,8 \% \quad 65,4 \% \quad 10,3 \%$

¿Consideras que la integración de las TIC

favorece el aprendizaje?

$3,7 \% \quad 41,1 \% \quad 40,2 \% \quad 15,0 \%$

¿Conoce las posibilidades didácticas de las

TIC en el aula?

¿Sabría integrar las TIC en el aula?

¿Considera que es competente tecnológicamente?

¿Se siente preparado para usar las TIC cuando trabaje de maestro?

$26,2 \% 43,0 \%$

$0 \% \quad 19,6 \% \quad 48,6 \% \quad 31,8 \%$

$9,3 \% \quad 24,3 \% \quad 32,7 \% \quad 33,6 \%$

$6,5 \% \quad 37,4 \% \quad 31,8 \% \quad 24,3 \%$

¿Cree que tiene una formación suficiente

para integrar las tecnologías en su posterior práctica docente?

En su periodo de prácticas, ¿ha utilizado las $\mathrm{TIC}$ ?

En su periodo de prácticas, ¿ssu tutor utilizaba las TIC?

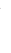

¿Relacionaría el uso de las TIC con la innovación docente?

¿En qué medida considera que es importante que los docentes estén alfabetizados tecnológicamente?

¿Considera difícil la incorporación de las TIC en las metodologías docentes?

¿Utiliza las TIC para establecer redes con sus compañeros $y / 0$ profesores?

$\begin{array}{llll}0,9 \% & 29,0 \% & 44,9 \% & 25,2 \%\end{array}$

¿En qué medida cree que las TIC mejora la enseñanza?

$13,1 \% 42,1 \% \quad 35,5 \%$

$9,3 \%$

$5,0 \% 29,0 \%$

$32,7 \%$

$23,4 \%$

$16,8 \% 19,6 \%$

$45,8 \%$

$17,8 \%$

$3,7 \% \quad 23,4 \%$

$45,8 \%$

$27,1 \%$

El uso de herramientas como las wikis o la webquest es un recurso de aprendizaje útil

$\begin{array}{llll}0 \% & 12,1 \% & 20,6 \% & 67,3 \%\end{array}$

$\begin{array}{llll}0 \% & 46,7 \% & 7,5 \% & 45,8 \%\end{array}$

$3,7 \% \quad 10,3 \% \quad 40,2 \% \quad 45,8 \%$

$3,7 \% \quad 14,0 \% \quad 39,3 \% \quad 43,0 \%$

El visionado de vídeos y juegos interactivos favorece la motivación del alumnado

$29,9 \% \quad 3,7 \% \quad 29,0 \% \quad 37,4 \%$ 
La competencia científico-tecnológica en la formación del futuro docente: algunos aspectos de la autopercepción con respeto a la integración de las TIC en el aula Pedro Tadeu

\begin{tabular}{|c|c|c|c|c|}
\hline Ítem & Nada & Poco & Bastante & Mucho \\
\hline $\begin{array}{l}\text { Las actividades interactivas mejoran el apren- } \\
\text { dizaje del alumnado }\end{array}$ & $36,4 \%$ & $44,9 \%$ & $2,8 \%$ & $15,9 \%$ \\
\hline $\begin{array}{l}\text { El diseño de actividades con las TIC dificulta } \\
\text { la labor docente }\end{array}$ & $2,8 \%$ & $24,3 \%$ & $36,4 \%$ & $36,4 \%$ \\
\hline $\begin{array}{l}\text { El uso de las TIC facilita la evaluación del } \\
\text { alumnado }\end{array}$ & $0 \%$ & $18,7 \%$ & $63,6 \%$ & $17,8 \%$ \\
\hline $\begin{array}{l}\text { El uso de las TIC contribuye la construcción } \\
\text { de aprendizajes significativos }\end{array}$ & $0 \%$ & $22,4 \%$ & $62,6 \%$ & $15,0 \%$ \\
\hline $\begin{array}{l}\text { Las TIC ayudan a conseguir una mejor inte- } \\
\text { gración de los alumnos en la sociedad }\end{array}$ & $0 \%$ & $22,4 \%$ & $43,9 \%$ & $33,6 \%$ \\
\hline $\begin{array}{l}\text { El uso de dispositivos móviles son un recurso } \\
\text { útil para que el alumnado aprenda }\end{array}$ & $0 \%$ & $0 \%$ & $31,8 \%$ & $68,2 \%$ \\
\hline $\begin{array}{l}\text { Considera que las TIC son adecuadas en los } \\
\text { procesos de enseñanza y aprendizaje con } \\
\text { alumnado con NEE }\end{array}$ & $0 \%$ & $18,7 \%$ & $19,6 \%$ & $61,7 \%$ \\
\hline Las TIC contribuyen a la inclusión & $0 \%$ & $0 \%$ & $54,2 \%$ & $45,8 \%$ \\
\hline $\begin{array}{l}\text { Por lo general, ¿consideras que el profesora- } \\
\text { do usa las TIC correctamente? }\end{array}$ & $0 \%$ & $3,7 \%$ & $69,2 \%$ & $27,1 \%$ \\
\hline $\begin{array}{l}\text { Por lo general, ¿Consideras que se hace un } \\
\text { uso adecuado de las herramientas tecnológi- } \\
\text { cas en la escuela? }\end{array}$ & & & $29,9 \%$ & $0,9 \%$ \\
\hline $\begin{array}{l}\text { Por lo general, ¿Consideras que el profesora- } \\
\text { do tiene la formación suficiente para usar las } \\
\text { TIC correctamente? }\end{array}$ & & & $16,8 \%$ & $0,9 \%$ \\
\hline $\begin{array}{l}\text { Por lo general, ¿Consideras que las TIC son } \\
\text { útiles en educación? }\end{array}$ & $0 \%$ & $8,4 \%$ & $30,8 \%$ & $60,7 \%$ \\
\hline
\end{tabular}

Antes de cruzar las variables, reagrupamos las respuestas para obtener las respuestas de cada pregunta: nada/poco y bastante/mucho, es decir, transformamos las variables en dicotomías.

Aplicando el test de independencia chi-cuadrado, encontramos que las respuestas obtenidas a "En qué medida cree que las TIC mejora la enseñanza" estaban estadísticamente asociadas a las respuestas obtenidas en: "Consideras que la integración de las TIC favorece el aprendizaje" $(\chi=21,444 ; \rho<.001)$, "Conoce las posibilidades didácticas de las TIC en el aula" $(\chi=31,901 ; \rho<.001)$ y "Sabría integra las TIC en el aula" $(\chi=34,407 ; \rho<.001)$. Se observó que cuanto más valoramos las TIC en la enseñanza, más reconocemos la importancia de las TIC en el aprendizaje, más conocemos las posibilidades de enseñanza de las TIC y más sabemos cómo integrarlas en clase. 
Aplicando el test de independencia chi-cuadrado, se concluyó que "el uso de las TIC contribuye a la construcción de aprendizajes significativos" y "las TIC ayudan a lograr una mejor integración de los estudiantes en la sociedad" se asociaron de manera significativa: "Considera que la integración de las TIC favorece el aprendizaje" ( $\chi=38.030 ; \rho<.001)$, "Conoce las posibilidades didácticas de las TIC en el aula" $(\chi=7.555)$; $\rho<.01)$, "Me siento preparado para utilizar las TIC cuando trabaje como profesor " $(\chi=13.797 ; \rho<.001)$, " Crees que tienes la formación suficiente para integrar las tecnologías en tu práctica docente " $(\chi=20.713 ; \rho<.001)$.

La aplicación de la prueba exacta de Fisher concluyó que "Por lo general, ¿Consideras de que las TIC son útiles en la educación?" se asoció significativamente con: "¿Consideras que la integración de las TIC favorece el aprendizaje?" ( $\chi=7.994 ; \rho<.01)$ y “¿Sabría Integrar las TIC en el aula?" $(\chi=4.982 ; \rho<.05)$.

Aplicando la prueba exacta de Fisher se concluyó que "Por lo general, ¿Consideras que las TIC son útiles en la educación?" es estadísticamente independiente de "Por lo general, ¿Consideras que el profesor utiliza correctamente las $\mathrm{TIC}^{\prime}$; " Por lo general, ¿Consideras que hay un uso adecuado de las herramientas tecnológicas en la escuela?" y " Por lo general, ¿Consideras que el profesor tiene suficiente formación para utilizar correctamente las TIC?". Por lo tanto, podemos afirmar que en este estudio la opinión de los futuros profesores sobre la utilidad de las TIC en la educación no está asociada a su opinión sobre el uso correcto de las TIC y las herramientas tecnológicas de la escuela, ni a la opinión sobre la formación en el uso de las TIC.

Los resultados obtenidos para "¿Considera difícil incorporar las TIC en los métodos de enseñanza?" fueron estadísticamente independientes de "Por lo general, ¿Consideras que se hace un uso adecuado de las herramientas tecnológicas en la escuela?" por la prueba de Chi-cuadrado. Podemos afirmar que la dificultad de incorporar las TIC en las metodologías de enseñanza no depende del uso correcto de las tecnologías existentes en las escuelas.

\section{Discusión y Conclusiones}

La incorporación de las TIC al ámbito educativo ha transformado los procesos de enseñanza y aprendizaje, situando a los docentes en una 
posición compleja. En la actualidad, se busca la incorporación de las TIC en los procesos instructivos, fruto de procesos reflexivos en los que se conjugue el conocimiento técnico de los recursos (alfabetización tecnológica) y el conocimiento pedagógico necesario, para utilizar la tecnología, usar los recursos más adecuados para cada concepto y "conocimiento susceptible de ser enseñado", a fin de asentar las bases para la construcción de nuevos conocimientos (Wong, 2015). En esta línea, conocer y dominar los recursos tecnológicos, es decir, el conocimiento meramente técnico, es insuficiente para incluir eficazmente las TIC en la tarea docente.

En esta línea, se sitúan los trabajos de Koehler y Mishra (2009) o el de Koehler, Mishra y Cain (2015), quiénes van un paso más allá, asegurando que la formación del profesorado no debe atender exclusivamente a cómo utilizar la tecnología, sino que debería proporcionar un conocimiento integrado en el que se conjugasen las posibilidades didácticas de la tecnología, con el conocimiento pedagógico y el contenido susceptible de ser enseñado, denominado TPACK. Este paradigma está adquiriendo gran importancia en la actualidad. De hecho, gran parte de los trabajos empíricos que abarcan la cuestión de la incorporación del TIC en los procesos instructivos de forma eficaz, atienden a las directrices y presupuestos evidenciados en él, conjugando los tres elementos básicos asumidos: conocimiento tecnológico, de contenido y pedagógico (Cabero y Barroso, 2016).

En el estudio desarrollado con estudiantes de enseñanza básica del IPG, se encontró que los aspirantes a docentes conocían las posibilidades didácticas ofrecidas por las TIC, asegurando que su uso facilita los procesos instruccionales. Estos resultados discrepan de los estudios de Gabarda-Méndez, Rodríguez-Martín y Moreno-Rodríguez (2017), donde se evidencia las dificultades que tienen los aspirantes a docentes para "identificar necesidades y recursos digitales, tomar decisiones a la hora de elegir la herramienta digital apropiada, acorde a la finalidad o necesidad, resolver problemas conceptuales a través de medios digitales, resolver problemas técnicos, el uso creativo de la tecnología" (p. 265). En esta línea, también se encuentran los trabajos de Cózar, Valle, Hernández y Hernández (2015), que ponen de relieve las dificultades del profesorado para utilizar las TIC debido a sus deficiencias formativas y el acceso a los recursos tecnológicos.

El presente estudio fue desarrollado en el marco de una asignatura 
connectada con la Educación multimedia, donde los estudiantes aprendieron a utilizar diferentes recursos y herramientas tecnológicas educativas. En el transcurso del mismo y en vista a los resultados obtenidos, los participantes del estudio afirmaban ser competentes en tecnología, coincidiendo con investigaciones halladas en la literatura (Almerich, Orellana y Díaz-García, 2015; Gallego-Arrufat, Gámiz-Sánchez y Gutiérrez-Santiuste, 2010). Sin embargo y en sintonía con un gran corpus de estudios, no se sienten lo suficientemente preparados para incorporar la tecnología a su ejercicio docente, señalando ciertas carencias formativas que limitan su puesta en práctica (Passey et al., 2018). Otros estudios han señalado a la predisposición positiva del profesorado hacia las TIC (Luan y Teo, 2011) y su confianza para incorporarlas en sus prácticas docentes como el principal predictor de uso de las mismas (Sang, Valcke, Braak y Tondeur, 2010).

Al respecto, estudios como los llevados a cabo por Bagetti, Mussoi y Mallmann (2017), abogan por el uso de Recursos Educativos Abiertos (REA) para asegurar la fluidez tecnológico-pedagógica. En su estudio encontraron que el diseño de video aula con recursos REA, favorecía la construcción de aprendizajes significativos en el alumnado, al tiempo que eran especialmente útiles para asegurar una capacitación tecnológica docente, que conjugase los componentes tecnológicos y pedagógicos en su formación inicial.

Además, la totalidad de los participantes relaciona las TIC con los procesos de innovación, por lo que le otorgan gran importancia al hecho de que los docentes posean competencias tecnológicas. Asimismo, al preguntarles si ven factible la incorporación de las TIC en la práctica docente, consideran que es una tarea compleja. Algunos estudios como el desarrollado por Rioseco-Pais, Paukner-Nogués y Ramírez-Muñoz (2017) también ha dado cuenta de la disyuntiva entre la actitud hacia las TIC y el uso de herramientas tecnológicas con estudiantes universitarios. En estés estudios fueron analizados el progreso tecnológico y las actitudes hacia el aprendizaje de estudiantes de pedagogía a partir de una actividad curricular denominada "Powtown", esta es una herramienta educativa online para la creación de animaciones y todo tipo de presentaciones en video. En sus resultados encontraron que los participantes consideraban que esta actividad le había permitido adquirir nuevas habilidades, era una herramienta adecuada para aprender nuevos contenidos ya que actuaba de soporte para profesores y alumnos, a la vez que 
La competencia científico-tecnológica en la formación del futuro docente: algunos aspectos de la autopercepción con respeto a la integración de las TIC en el aula Pedro Tadeu

despertaba su motivación y creatividad. Supeditado también a la innovación es el estudio longitudinal realizado por Sotiriou, Riviou, Cherouvis, Chelioti y Bogner (2016). Contextualizado en el programa europeo Open Discovery Space (ODS), analiza los efectos positivos que tuvo una metodología innovadora basada en la tecnología para la alfabetización tecnológica del profesorado, la colaboración profesional docente y la familiarización con el uso de recursos tecnológicos y digitales, en nuestro estudio se verifica conexiones con este programa europeo en cuanto a los aspectos positivos del uso de nuevas e innovadoras metodologías basadas en tecnología (86\% de los alumnos valora que las TIC mejoran la enseñanza en Bastante y Mucho).

Respecto a la atención a la diversidad, y conectando a los resultados de las cuestiones aplicadas ( $i$ Considera que las TIC son adecuadas en los procesos de enseñanza y aprendizaje con alumnado con NEE?; $i$ Las TIC contribuyen a la inclusión?), los participantes afirman que las TIC favorece la inclusión educativa $(100 \%$ de los alumnos consideran Bastante y Mucho en sus respuestas), pues ofrecen herramientas adecuadas para el alumnado con necesidades educativas especiales (un total de $81,3 \%$ se divide entre Bastante y Mucho). Este hallazgo es consistente con la literatura especializada en el tema, que ha establecido fuertes nexos de unión entre la tecnología educativa y la Educación Especial. Ejemplo de ello son los trabajos de Istenic y Bagon (2014), que aporta una revisión sistemática de los trabajos que han unificado el uso de las TIC y la Educación Especial o el aporte de Hersh (2017), quién ofrece una clasificación de herramientas tecnológicas "inclusivas" para el alumnado con discapacidad.

Asimismo, se asocia una mayor valoración de las TIC en la enseñanza, junto con una valoración positiva en cuanto a la importancia de las TIC en el proceso de enseñanza-aprendizaje. Además, la valoración positiva conlleva que los futuros docentes reconozcan las posibilidades que ofrece su integración en el aula. Esta relación lógica ha sido identificada en investigaciones como Luan y Teo (2011), en las que asocian la predisposición hacia las TIC con la edad, en favor de los más jóvenes, al ser más proclives al uso de la tecnología.

Un aspecto sorprendente hallado en este estudio es que la dificultad de incorporar las TIC en las metodologías de enseñanza no depende del uso correcto de las tecnologías existentes en las escuelas. Este hallazgo discrepa de la mayoría de las investigaciones existentes sobre el tema 
La competencia científico-tecnológica en la formación del futuro docente: algunos aspectos de la autopercepción con respeto a la integración de las TIC en el aula

Pedro Tadeu

(George y Sanders, 2017; García-Valcárcel Muñoz-Repiso y Martín del Pozo, 2016).

Asimismo, la percepción de los futuros profesores sobre la utilidad de las TIC en la educación no está asociada con su percepción sobre el uso correcto de las TIC y las herramientas tecnológicas de la escuela o la relacionada con la formación en el uso de las TIC. Es decir, los aspirantes a docentes consideran que las TIC son útiles para su posterior ejercicio profesional, pero no relacionan su efectividad a la capacitación tecnológica o a la formación recibida en tecnologías. Esto discrepa de investigaciones como la de Méndez-Garrido y Delgado-García (2016), en las que señalan el acceso a los recursos y las deficiencias formativas como principales limitaciones para la integración adecuada de las TIC en las escuelas de primaria e infantil.

Pese a señalar las limitaciones que conllevan, especialmente una falta de cultura tecnológica entre el profesorado en ejercicio, sumado a una formación insuficiente en algunos casos, los futuros docentes reconocen la utilidad de las nuevas tecnologías en el ámbito de educación.

En este sentido, parece que aún queda camino por recorrer en el logro de una integración efectiva de las TIC en los centros educativos. Sin duda, el inicio debe pasar por una capacitación tecnológica docente, tanto en su etapa inicial como en los programas de formación docente, que doten al profesorado del conocimiento y herramientas necesarias para profesionalizar su figura y, por ende, sus prácticas en el aula.

\section{Referencias}

Almerich, G., Orellana, N. y Díaz-García, I. (2015). Las competencias en TIC en el profesorado en formación y su relación con las creencias pedagógicas, la autoeficacia y la percepción del impacto de las TIC en la educación. Investigar con y para la sociedad, 2, 589-598.

Bagetti, S., Mussoi, E.M. y Mallmann, E.M. (2017). Fluência tecnológico-pedagógica na produção de recursos educacionais abertos-REA. Texto Livre: Linguagem e Tecnologia, 10(2), 185-205.

Cabero, J. y Barroso, J. (2016). ICT teacher training: a view of the TPACK model. Culture and Education, 28(3), 633-663.Doi: 10.1080/11356405.2016.1203526

Casey, A., Goodyear, V.A. y Armor, K. (2017). Digital technologies and learning in physical education: pedagogical cases. United Kingdom: Routledge

Cózar, R., Valle, M. D., Hernández, J. A. y Hernández, J. R. (2015). Tecnologías emergentes para la enseñanza de las ciencias sociales: Una experiencia con el uso de realidad 
La competencia científico-tecnológica en la formación del futuro docente: algunos aspectos de la autopercepción con respeto a la integración de las TIC en el aula Pedro Tadeu

aumentada en la formación inicial de maestros. Digital Education Review, 27, 138153. Doi:10.1344/der.2015.27.138-153

De-Juanas Oliva, Á., Martín-del Pozo, R. y González-Ballesteros, M. (2016). Competencias docentes para desarrollar la competencia científica en educación primaria. Bordón. Revista de Pedagogía, 68(2), 103-120. Doi:10.13042/bordon.2016.68207

Fernández-Batanero, J.M., Cabero, J. y López-Meneses, E. (2018). Knowledge and degree of training of primary education teachers in relation to ICT taught to students with disabilities. British Journal of Educational Technology. Volume 50, Issue 4, Doi:10.1111/ bjet.12675

Fernández-Batanero, J. M., Sañudo, B., Montenegro-Rueda, M. y García-Martínez, I. (2019). Physical Education Teachers and Their ICT Training Applied to Students with Disabilities. The Case of Spain. Sustainability, 11(9), 2559. Doi:10.3390/su11092559

Ferrari, A. (2013). DIGCOMP. A framework for Developing and Understanding Digital Competence in Europe. Sevilla. Doi: 10.2788/52966.

Gabarda-Méndez, V., Rodríguez-Martín, A. y Moreno-Rodríguez, M. D. (2017). La competencia digital en estudiantes de magisterio. Análisis competencial y percepción personal del futuro maestro. Educatio Siglo XXI, 35, 253-274. Doi:10.6018/j/298601.

García-Pérez, R., Rebollo-Catalán, Á. y García-Pérez, F. (2016). Relación entre las preferencias de formación del profesorado y su competencia digital en las redes sociales. Bordón. Revista de Pedagogía, 68(2), 137-153. Doi:10.13042/bordon.2016.68209.

García-Valcárcel Muñoz-Repiso, A. y Martín del Pozo, M. (2016). ¿Se sienten preparados los graduados en maestro de primaria para afrontar la profesión docente? Bordón. Revista de Pedagogía, 68 (2), 69-84. Doi:10.13042/bordon.2016.68205

George, A. y Sanders, M. (2017). Evaluating the potential of teacher-designed technology-based tasks for meaningful learning: Identifying needs for professional development. Education and Information Technologies, 24(6), 2871-2895. Doi:10.1007/ s10639-017-9609-y

Gisbert-Cervera, M., González-Martínez, J. y Esteve-Mon, F. M. (2016). Competencia digital y competencia digital docente: una panorámica sobre el estado de la cuestión. Revista Interuniversitaria de Investigación en Tecnología Educativa, 0, 74-83. Doi: 10.6018/riite/2016/257631.

Istenic, A. y Bagon, S. (2014). ICT supported learning for inclusion of people with special needs: review of seven educational technology journals, 1970-2011. British Journal of Educational Technology, 45(2), 202-230. Doi:10.1111/bjet.12086

Hersh, M. (2017). Classification framework for ICT-based learning technologies for disabled people. British Journal of Educational Technology, 48(3), 768-788. Doi:10.1111/ bjet. 12461

Khine, M., Afari, E. y Ali, N. (2019). Investigating Technological Pedagogical Content Knowledge Competencies among Trainee Teachers in the Context of ICT Course. Alberta Journal of Educational Research, 65(1), 22-36.

Koehler, M. y Mishra, P. (2009). What is technological pedagogical content knowledge (TPACK)? Contemporary issues in technology and teacher education, 9(1), 60-70.

Koehler, M. J., Mishra, P. y Cain, W. (2015). ¿Qué son los saberes tecnológicos y pedagógicos del contenido (TPACK)? Virtualidad, educación y ciencia, 6(10), 9-23. 
La competencia científico-tecnológica en la formación del futuro docente: algunos aspectos de la autopercepción con respeto a la integración de las TIC en el aula

Pedro Tadeu

Liu, G.Z., Wu, N.W. y Chen, Y.W. (2013). Identifying emerging trends for implementing learning technology in special education: A state-of-the-art review of selected articles published in 2008-2012. Research in developmental disabilities, 34(10), 3618-3628. Doi: 10.1016/j.ridd.2013.07.007.

López, M. C., Pérez-García, P. y Rodríguez, M. J. (2015). Concepciones del profesorado universitario sobre la formación en el marco del espacio europeo de educación superior. Revista de Investigación Educativa, 33(1), 179-194. Doi: 10.6018/ rie.33.1.189811.

Luan, W. S. y Teo, T. (2011). Student Teachers' Acceptance of Computer Technology: An Application of the Technology Acceptance Model (TAM). En Timothy Teo (Ed.) Technology Acceptance in Education: Research and Issues (pp. 43-61). Rotterdam: SensePublishers.

Magen-Nagar, N. y Shonfeld, M. (2018). Attitudes, openness to multiculturalism, and integration of online collaborative learning. Journal of Educational Technology \& Society, 21(3), 1-11.

Martínez, R., Leite, C., y Monteiro, A. (2016). TIC y formación inicial de maestros: oportunidades y problemas desde la perspectiva de los estudiantes. Cuadernos de Investigación Educativa, 7(1), 69-92. Doi: 10.18861/cied.2016.7.1.2577.

Méndez Garrido, J. M., y Delgado-García, M. (2016). ICT in Primary and Secondary Education Schools in Andalusia. A case study from best practices. Digital Education Review, (29), 134-165. Doi:10.1344/der.2016.29.134-165.

Passey, D., Shonfeld, M., Appleby, L., Judge, M., Saito, T. y Smits, A. (2018). Digital agency: Empowering equity in and through education. Technology, Knowledge and Learning, 23(3), 425-439. https://doi.org/10.1007/s10758-018-9384-x

Rioseco-Pais, M. H., Paukner-Nogués y Ramírez-Muñoz, B. (2017). Incorporating Powtoon as a Learning Activity into a Course on Technological Innovations as Didactic Resources for Pedagogy Programs. International Journal of Emerging Technologies in Learning, 12(6), 120-131. Doi: 10.3991/ijet.v12i06.7025.

Sánchez-García, A. B. y Galindo-Villardón, P. (2018). Uso e integración de las tic en el aula y dificultades del profesorado en activo de cara a su integración. Profesorado, Revista de Currículum y Formación del Profesorado, 22(3), 341-358.

Sánchez-Prieto, J.C., Hernández-García, Á., García-Peñalvo, F. J., Chaparro-Peláez, J. y Olmos-Migueláñez, S. (2019). Break the walls! Second-Order barriers and the acceptance of mLearning by first-year pre-service teachers. Computers in Human Behavior, 95, 158-167. Doi: 10.1016/j.chb.2019.01.019.

Sang, G., Valcke, M., Braak, J. V. y Tondeur, J. (2010). Student teachers' thinking processes and ICT integration: Predictors of prospective teaching behaviors with educational technology. Computers \& Education, 54(1), 103-112. Doi: 10.1016/j.compedu.2009.07.010.

Sotiriou, S., Riviou, K., Cherouvis, S., Chelioti, E., y Bogner, F. X. (2016). Introducing large-scale innovation in schools. Journal of Science Education and Technology, 25(4), 541-549. Doi: 10.1007/s10956-016-9611-y.

UNESCO. (2008). ICT competency standards for teachers. Policy Framework (156210). Paris, France: United Nations Educational, Scientific and Cultural Organization. 
La competencia científico-tecnológica en la formación del futuro docente: algunos aspectos de la autopercepción con respeto a la integración de las TIC en el aula Pedro Tadeu

Wong, G.K.W. (2015). Understanding Technology Acceptance in Pre-service teachers of primary mathematics in Hong Kong. Australasian Journal of Educational Technology, 31(6), 713-735. Doi: 10.14742/ajet.1890.

\section{Agradecimientos}

Este trabalho é financiado por Fundos Nacionais através da FCT - Fundação para a Ciência e a Tecnologia, I.P., no âmbito do projeto Refa UIDB/05507/2020. Agradecemos adicionalmente ao Centro de Estudos em Educação e Inovação (Cl\&DEI) e ao Politécnico da Guarda pelo apoio prestado. 